\title{
Ultrafast vectorial and scalar dynamics of ionic clusters: Azobenzene solvated by oxygen
}

\author{
D. Hern Paik, J. Spencer Baskin, Nam Joon Kim, ${ }^{\text {a) }}$ and Ahmed H. Zewail ${ }^{\text {b) }}$ \\ Laboratory for Molecular Sciences, California Institute of Technology, Pasadena, California 91125 \\ and Physical Biology Center for Ultrafast Science and Technology, California Institute of Technology, \\ Pasadena, California 91125
}

(Received 24 February 2006; accepted 20 April 2006; published online 5 October 2006)

\begin{abstract}
The ultrafast dynamics of clusters of trans-azobenzene anion $\left(A^{-}\right)$solvated by oxygen molecules was investigated using femtosecond time-resolved photoelectron spectroscopy. The time scale for stripping off all oxygen molecules from $A^{-}$was determined by monitoring in real time the transient of the $A^{-}$rise, following an $800 \mathrm{~nm}$ excitation of $A^{-}\left(\mathrm{O}_{2}\right)_{n}$, where $n=1-4$. A careful analysis of the time-dependent photoelectron spectra strongly suggests that for $n>1$ a quasi- $\mathrm{O}_{4}$ core is formed and that the dissociation occurs by a bond cleavage between $A^{-}$and conglomerated $\left(\mathrm{O}_{2}\right)_{n}$ rather than a stepwise evaporation of $\mathrm{O}_{2}$. With time and energy resolutions, we were able to capture the photoelectron signatures of transient species which instantaneously rise ( $<100 \mathrm{fs})$ then decay. The transient species are assigned as charge-transfer complexes: $A \cdot \mathrm{O}_{2}^{-}$for $A^{-} \mathrm{O}_{2}$ and $A \cdot \mathrm{O}_{4}^{-} \cdot\left(\mathrm{O}_{2}\right)_{n-2}$ for $A^{-}\left(\mathrm{O}_{2}\right)_{n}$, where $n=2-4$. Subsequent to an ultrafast electron recombination, $A^{-}$rises with two distinct time scales: a subpicosecond component reflecting a direct bond rupture of the $A^{-}-\left(\mathrm{O}_{2}\right)_{n}$ nuclear coordinate and a slower component (1.6-36 ps, increasing with $n)$ attributed to an indirect channel exhibiting a quasistatistical behavior. The photodetachment transients exhibit a change in the transition dipole direction as a function of time delay. Rotational dephasing occurs on a time scale of 2-3 ps, with a change in the sign of the transient anisotropy between $A^{-} \mathrm{O}_{2}$ and the larger clusters. This behavior is a key indicator of an evolving cluster structure and is successfully modeled by calculations based on the structures and inertial motion of the parent clusters. (C) 2006 American Institute of Physics. [DOI: 10.1063/1.2205855]
\end{abstract}

\section{INTRODUCTION}

Spectroscopy on mass-selected anionic clusters has been recognized as a versatile tool to carry out systematic studies of stepwise solvation. ${ }^{1-7}$ Rigorous mass selection allows us to follow the evolution of size-dependent properties to bulk and to examine the solvation dynamics as a function of cluster size. In earlier works, we have shown that these aspects appeared in homogeneous and heterogeneous anionic oxygen clusters. ${ }^{8,9}$ In continuation of our efforts on oxygen-solvated anionic clusters, we have performed femtosecond timeresolved photoelectron (PE) spectroscopy experiments to study the photodissociation of anionic clusters of transazobenzene anion $\left(A^{-}\right)$solvated by oxygen molecules. In this contribution, we studied the photodetachment of the isolated $A^{-}$and its oxygen clusters and provided photochemical data for $A^{-}\left(\mathrm{O}_{2}\right)_{n}$, where $n=0-4$, in gas phase, including photoelectron spectra and photofragment patterns. Our focus is on the dissociation dynamics of these finite-sized clusters taking place on the femtosecond time scale.

In our experiment, we excite $A^{-}\left(\mathrm{O}_{2}\right)_{n}$ with an $800 \mathrm{~nm}$ femtosecond pulse and detect photoelectrons detached by a $400 \mathrm{~nm}$ femtosecond pulse. With the pump-probe scheme, the bond dissociation between $A^{-}$and $\left(\mathrm{O}_{2}\right)_{n}$ was monitored

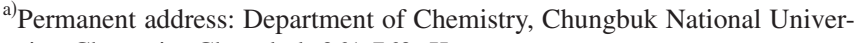
sity, Cheongju, Chungbuk 361-763, Korea.

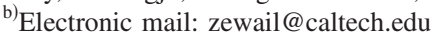

in real time by changing the time delay between the two pulses. The observed biexponential rise of $A^{-}$in our transients, interpreted in light of the time-dependent PE spectra, provides key elements of the dynamics. We provide experimental evidence that the dissociation occurs via electron transfer followed by an ultrafast electron recombination and revisit the critical role of $\mathrm{O}_{2}$ as an electron acceptor in photoinduced electron transfer.

In this study, we also explore the potential application of femtosecond time-resolved anisotropy as a means to elucidate the geometry of clusters. Time-resolved anisotropy has been developed as a reliable method to determine molecular structures and monitor the vectorial dynamics of rotation. ${ }^{10-12}$ We applied this technique to study the structure and rotational motion of $A^{-}\left(\mathrm{O}_{2}\right)_{n}$ clusters and deduced a change in the location of $\left(\mathrm{O}_{2}\right)_{n}$ with respect to $A^{-}$upon going from $A^{-} \mathrm{O}_{2}$ to $A^{-} \mathrm{O}_{4}$. The cluster effect on the rotational motion is examined by measuring the dephasing time of the initial alignment. From the trend observed in the polarization dependence, we deduce the direction of the transition dipole moment for the photodetachment (probe) process.

\section{EXPERIMENT}

The molecular beam apparatus and the femtosecond laser system have been described elsewhere. ${ }^{13,9}$ Briefly, negatively charged $A_{m}^{-}\left(\mathrm{O}_{2}\right)_{n}$ clusters were generated by crossing a $1.0 \mathrm{keV}$ electron beam with a supersonic expansion of 
(A)

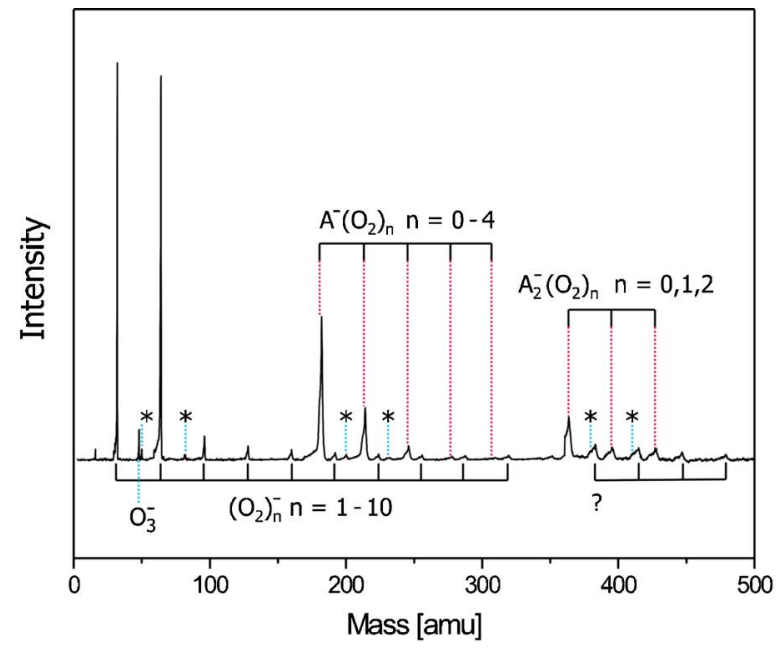

(B)

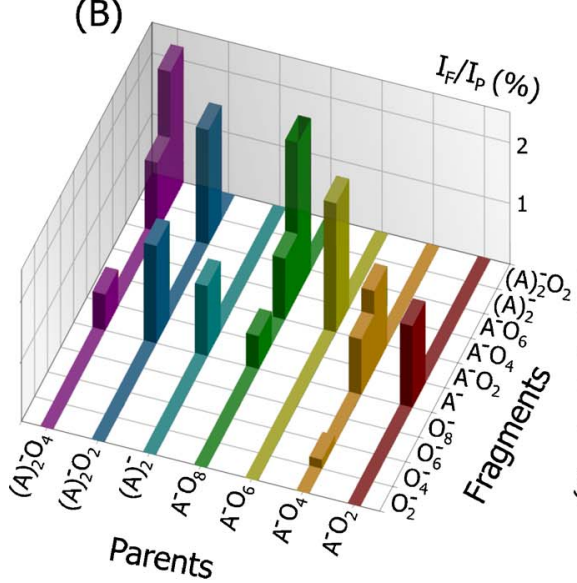

(C)

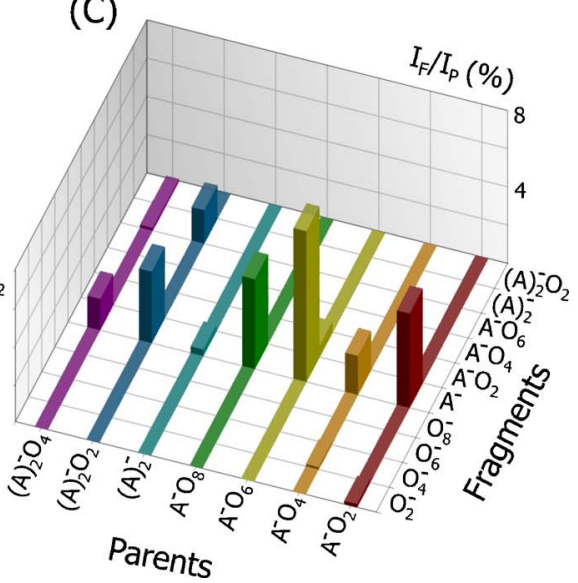

FIG. 1. (Color online) (A) Mass spectrum of $A_{m}^{-}\left(\mathrm{O}_{2}\right)_{n}$ generated by the pulsed electron impact source. The traces of $\left(\mathrm{O}_{2}\right)_{n}^{-} \mathrm{H}_{2} \mathrm{O}, A_{m}^{-}\left(\mathrm{H}_{2} \mathrm{O}\right)$, and $A_{m}^{-} \mathrm{O}_{2}\left(\mathrm{H}_{2} \mathrm{O}\right)$ clusters are indicated by the asterisk. An unidentified ion peak at mass $=383 \mathrm{amu}$ and its water clusters are indicated by a question mark (see text for details). (B) Metastable species detected by the linear reflectron time-of-flight mass spectrometer (without a laser pulse). The detection threshold in the $A^{-} \mathrm{O}_{6}$ experiment was lower than for other clusters due to a lower signal level. (C) Photofragments generated by irradiating with the pump pulse $(800 \mathrm{~nm})$. For (B) and (C), the vertical axis corresponds to the fragment ion intensity and the enhancement of the ion intensity, respectively, $\left(I_{F}\right)$ normalized to the intensity $\left(I_{P}\right)$ of the stable parent ion (without laser). azobenzene/oxygen mixtures. The azobenzene $/ \mathrm{O}_{2}$ gas mixture was prepared by passing the oxygen gas $(400 \mathrm{kPa})$ through a sample oven containing azobenzene (Aldrich, $98 \%$ ) vapor at $100{ }^{\circ} \mathrm{C}$. A pulsed electric field $(-2.0 \mathrm{kV})$ was applied to the accelerator, and negatively charged species were extracted into the field free time-of-flight region where the negative ion bunches were separated by their masses. Mass selection of a particular ion packet was achieved by applying a pulsed electric field $( \pm 500 \mathrm{~V})$ to the interleaved mass gate ${ }^{14}$ prior to the light interaction. The desired species were then intercepted with femtosecond laser pulses in a perpendicular configuration, and photoelectrons and photofragments were generated. The photoelectrons were collected by a magnetic-bottle ${ }^{15}$ photoelectron spectrometer, while the photofragments were detected by a linear reflectron mass spectrometer. Transients were recorded by integrating the photoelectron intensity in a selected time-of-flight window.

Femtosecond pulses $(800 \mathrm{~nm} / 110 \mathrm{fs})$ from a Ti: $\mathrm{Al}_{2} \mathrm{O}_{3}$ oscillator were amplified to $5 \mathrm{~mJ}$ by a regenerative and a two-stage multipass amplifier at $20 \mathrm{~Hz}$. The $800 \mathrm{~nm}$ femtosecond pulse was frequency doubled by passing the light through a beta-barium borate (BBO) crystal. The residual $800 \mathrm{~nm}$ femtosecond light was used for the pump pulse, while the $400 \mathrm{~nm}$ doubled output was used for the probe. A time delay between pump and probe pulses was implemented by a computer-controlled translation stage. Typical pulse energies of the 800 and $400 \mathrm{~nm}$ pulses at the light-interaction region were 1.1 and $0.4 \mathrm{~mJ}$, respectively. The beams were collimated down to a diameter of $4.0 \mathrm{~mm}$ in order to achieve the optimal overlap between the molecular beam and light pulses without causing multiphoton processes. For timedependent anisotropy experiments, the polarization of the pump pulse was rotated with respect to the polarization of the probe pulse. For most experiments, the probe polarization was parallel to the photoelectron detection axis, but other configurations were tested and showed no effect on results.

The possibility of multiphoton processes was excluded based on the following studies of the pump-pulse power dependence. First, we observed a slope of somewhat less than 1 (about 0.7$)$ in the plot of $\log \left(I_{\text {signal }}\right)$ vs $\log \left(I_{\text {pump }}\right)$, consistent with a one-photon absorption of the $800 \mathrm{~nm}$ photon. ${ }^{16,17} \mathrm{Sec}-$ ond, we recorded transients at half the pump power and observed the same behavior. Accordingly, the transient signal reported here is for a one-photon excitation at $800 \mathrm{~nm}$. For the probe process, the kinetic energy value of the detached electron corresponds to the one-photon detachment at $400 \mathrm{~nm}$.

\section{RESULTS}

The mass spectrum of all anions created by the ion source is shown in Fig. 1(A). The mass peaks of $\left(\mathrm{O}_{2}\right)_{n}^{-} \mathrm{H}_{2} \mathrm{O}$ and $A_{m}^{-}\left(\mathrm{O}_{2}\right)_{n} \mathrm{H}_{2} \mathrm{O}$ are indicated by an asterisk. The broad fea- 
ture at a mass of $\sim 383$ amu is due to two unresolved peaks of masses of 382 and $383 \mathrm{amu}$, which were separated in the linear reflectron mass spectrometer (not shown). The mass peak of 383 amu (indicated by a question mark) and its progression by $32 \mathrm{amu}$ were not identified. The ion peaks of $A_{m}^{-}\left(\mathrm{O}_{2}\right)_{n}$ were relatively broad in comparison to $\left(\mathrm{O}_{2}\right)_{n}^{-}$peaks, indicating the presence of metastable species in the ion packets.

In experiments without laser light, negatively charged fragments from metastable species were separated and detected by the linear reflectron mass spectrometer. The intensity distribution of these fragments is presented in Fig. 1(B). The height of each bar shown in the histogram corresponds to the normalized intensity obtained by dividing the fragment ion intensity by the remaining parent ion intensity. The largest fragment component was $A^{-} \mathrm{O}_{6}$ generated from $A^{-} \mathrm{O}_{8}$ (2.6\%). No $\left(\mathrm{O}_{2}\right)_{n}^{-}$fragments were detected except for a very small amount $(0.1 \%)$ of $\mathrm{O}_{4}^{-}$generated from $A^{-} \mathrm{O}_{4}$, suggesting that the excess electron is localized in azobenzene, and the negatively charged azobenzene $\left(A^{-}\right)$is solvated by neutral $\mathrm{O}_{2}$ molecules.

Figure 1(C) shows the photofragment distribution produced upon irradiation with the $800 \mathrm{~nm}$ femtosecond pulse. The height of the histogram corresponds to the enhanced intensity of photofragments, which is obtained by subtracting the intensity due to metastable species in the absence of light, then dividing by the intensity of the parent ions used for Fig. 1(B). There are two noticeable features in Fig. 1(C): First, $A^{-}$is the major photofragment in all oxygen-containing clusters $(\sim 2 \%-8 \%)$. Second, no $\left(\mathrm{O}_{2}\right)_{n}^{-}$was detected except for small amounts of $\mathrm{O}_{2}^{-}(0.2 \%)$ and $\mathrm{O}_{4}^{-}(0.2 \%)$ generated from $A^{-} \mathrm{O}_{2}$ and $A^{-} \mathrm{O}_{4}$. In addition, when an oxygen molecule was not attached to $A^{-}$, e.g., $A_{2}^{-}[$in Fig. $1(\mathrm{C})]$ as well as $A_{3}^{-}$ (not shown), the photodissociation is almost negligible at $800 \mathrm{~nm}$.

Figure 2 shows the PE spectra of $A^{-}$and $A^{-}\left(\mathrm{O}_{2}\right)_{n}$ clusters obtained by irradiating with the probe pulse $(3.1 \mathrm{eV})$ only. The PE spectrum of $A^{-}$exhibits a broad Gaussian type profile with the onset [adiabatic electron affinity (AEA)] of $\sim 1 \mathrm{eV}$ and the peak [vertical detachment energy (VDE)] of about $\sim 1.6 \mathrm{eV}$. We did not observe any vibrational structure in the PE spectrum with our energy resolution $(\sim 250 \mathrm{meV})$. Our observed value for the AEA of $A^{-}$is about two times larger than the previously reported value $(0.56 \mathrm{eV})$ which was obtained from bracketing experiments. ${ }^{18,19}$ The discrepancy can be due to either a small Frank-Condon overlap at the 0-0 transition frequency, resulting in an overestimation of AEA from the photoelectron spectrum, or to the uncertainty in the entropy effect in the previous experiment. $^{18}$

The photoelectron spectrum of $A^{-} \mathrm{O}_{2}$ resembles the PE spectrum of the unsolvated $A^{-}$, but the spectrum is shifted toward higher electron binding energies (AEA $\sim 1.2 \mathrm{eV}$ and VDE $\sim 1.75 \mathrm{eV})$. The similarity and the small shift are evidence that the excess electron is localized in the azobenzene molecule and $\mathrm{O}_{2}$ is weakly bound to $A^{-}$. The PE spectra of larger clusters are similar to each other, but quite different from those of $A^{-}$and $A^{-} \mathrm{O}_{2}$. The PE spectra of $A^{-}\left(\mathrm{O}_{2}\right)_{n}$, where $n=2-4$, show broad bimodal profiles. The peak of

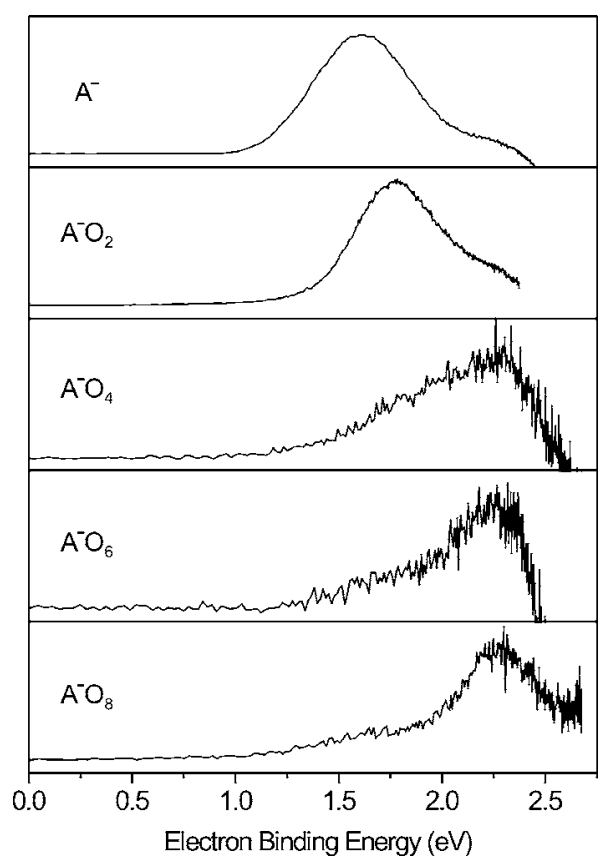

FIG. 2. Photoelectron spectra of $A^{-}\left(\mathrm{O}_{2}\right)_{n}$, where $n=1-4$, obtained by irradiating with the probe pulse $(3.1 \mathrm{eV})$.

each photoelectron spectrum is significantly shifted toward a higher electron binding energy $(\sim 2.3 \mathrm{eV})$ compared to that of $A^{-}$, while the onset is not much shifted $(\sim 1.25 \mathrm{eV})$. However, based on the metastable fragment pattern in Fig. 1(B), the region at the lower electron binding energy contains $\mathrm{PE}$ contributions from $A^{-}$and $A^{-} \mathrm{O}_{2}$ fragments. [The absence of these fragments for the $A^{-} \mathrm{O}_{6}$ parent in Fig. 1(B) could be due to low sensitivity. See the caption for details.] Even though the fragment population is small, the detachment cross sections of $A^{-}$and $A^{-} \mathrm{O}_{2}$ are larger then those of $A^{-}\left(\mathrm{O}_{2}\right)_{n}$, where $n=2-4$ (see below), so the fragment contribution to the spectra may be substantial, leading to artificially low AEA values. We note that the photoelectron collection efficiency is poorer for the slow electron [low electron kinetic energy (eKE) and high electron binding energy (eBE)]; thus the peak positions of $A^{-}\left(\mathrm{O}_{2}\right)_{n}$, where $n$ $=2-4$, are likely to be underestimated. Despite the abrupt change in the PE spectrum upon going from $A^{-} \mathrm{O}_{2}$ to $A^{-} \mathrm{O}_{4}$, we still think that the excess electron is localized in azobenzene. The abrupt change in the PE spectrum and in the relative photodetachment cross section is attributed to the formation of a quasi- $\mathrm{O}_{4}$ core (see Sec. IV).

The relative photodetachment cross sections $\left(\sigma_{\mathrm{PD}}\right)$ for $A^{-}\left(\mathrm{O}_{2}\right)_{n}$, where $n=0-4$, are roughly approximated from the ratio of the integrated ion peak [Fig. 1(A)] and the maximum intensity of the PE spectrum of the corresponding species (Fig. 2) which were obtained under similar experimental conditions. We assumed that the integrated area of the ion peak is proportional to the number density in the laser interaction volume, and we obtained a fourfold decrease in $\sigma_{\mathrm{PD}}$ for $A^{-} \mathrm{O}_{2}$ and $\sim 25$-fold for $A^{-}\left(\mathrm{O}_{2}\right)_{n}$, where $n=2-3$ compared to $A^{-}$. An equivalent estimate for $A^{-} \mathrm{O}_{8}$ cannot be made because its PE spectrum was obtained under different experimental conditions. Thus it is difficult to directly compare the 
(A)
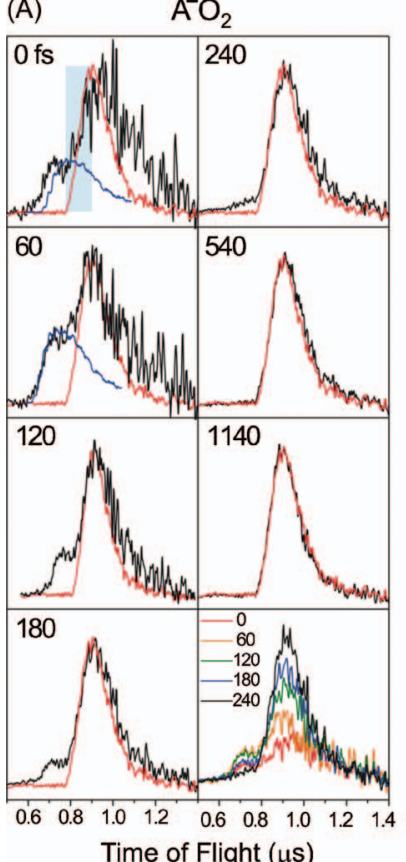

(B)

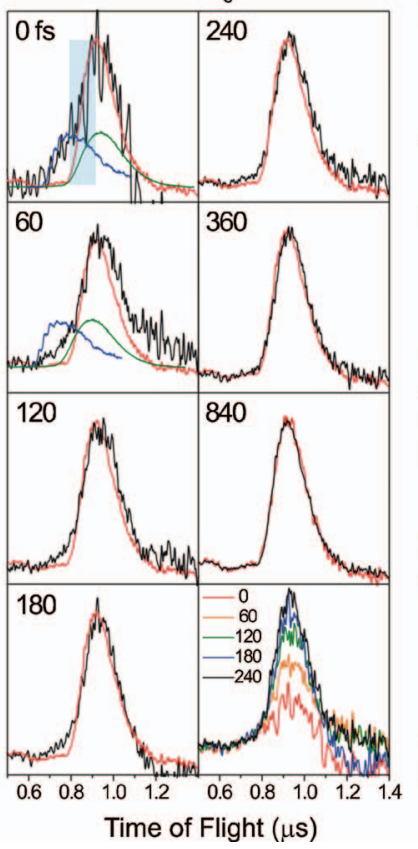

(C) TD-PE simulation

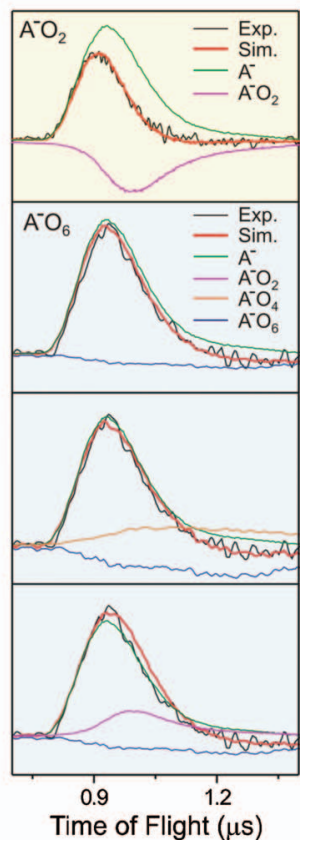

FIG. 3. (Color) (A) Time-dependent photoelectron spectra (TD-PE) of $A^{-} \mathrm{O}_{2}$ plotted on the electron time-of-flight axis. The label in each panel is the time delay in femtoseconds. Each spectrum is constructed by subtracting a reference (pump + probe at $t<0$ ) from a total signal (pump + probe at $t \geqslant 0$ ). In order to illustrate the change in shape, each panel, except for the bottom right, displays the TD-PE spectrum at the time delay indicated with intensity adjusted to match that of TD-PE at 83 ps (red trace). In the first and second panels, $\mathrm{PE}$ signals of $\mathrm{O}_{2}^{-}$and a vibrationally excited form of $\mathrm{O}_{2}^{-}$are overlaid with the TD-PE spectra of $A^{-} \mathrm{O}_{2}$ (see text). The bottom right panel contains a few TD-PE spectra in the true intensity scale as determined from the $A^{-} \mathrm{O}_{2}$ transient of Fig. 4. (B) TD-PE spectra of $A^{-} \mathrm{O}_{6}$. Same as (A), except that $\mathrm{PE}$ spectra of $\mathrm{O}_{4}^{-}$and vibrationally excited $\mathrm{O}_{4}^{-}$are shown in green and the intensities in the bottom right panel are determined from the $A^{-} \mathrm{O}_{6}$ transient of Fig. 4. (C) Simulated (red) and experimental (black) TD-PE spectra of $A^{-} \mathrm{O}_{2}$ (top panel) and $A^{-} \mathrm{O}_{2}$ (bottom three panels) at 83 ps. Second panel: simulation with $A^{-}$and $A^{-} \mathrm{O}_{6}$. Third panel: simulation with $A^{-}, A^{-} \mathrm{O}_{4}$, and $A^{-} \mathrm{O}_{6}$. Bottom panel: simulation with $A^{-}, A^{-} \mathrm{O}_{2}$, and $A^{-} \mathrm{O}_{6}$ (see text).

effect of $\sigma_{\mathrm{PD}}$ and the population of metastable fragments in the PE spectrum of $A^{-} \mathrm{O}_{8}$ to those of the other clusters.

Figure 3 shows the time-dependent photoelectron (TDPE) spectra of $A^{-} \mathrm{O}_{2}$ and $A^{-} \mathrm{O}_{6}$ that are constructed from the (pump+probe) signal at a delay time $t \geqslant 0$ minus the reference (pump+probe at $t<0$ ). Note that because ion fluctuation may make the measured reference either larger or smaller than the true reference of a given signal at $t \geqslant 0$, the uncertainty in regions of large parent PE intensity can be significant. The TD-PE spectra were obtained with the polarization of the pump pulse oriented at $54.7^{\circ}$ with respect to that of the probe pulse in order to suppress any effects of orientation dynamics. The spectra are presented on the timeof-flight (TOF) axis to emphasize the low energy region, and the peak intensity of each photoelectron spectrum is matched to that of the PE spectrum at $83 \mathrm{ps}$ (red trace) in order to illustrate the change in the peak shape with time. The bottom right panels of Figs. 3(A) and 3(B) show the overlay of a few TD-PE spectra in a true intensity scale as calibrated against recorded transients (see below). The time-delay labeling is also established by the transient analysis.

As seen here, and also for other clusters studied, the shape of the TD-PE spectrum does not change after $360 \mathrm{fs}$, matching that of the TD-PE spectrum at 83 ps. A peak corresponding to this long time TD-PE signature already appears at $0 \mathrm{fs}$ and continues to increase with time. At early times $(0-240 \mathrm{fs})$, the shapes of the TD-PE spectra differ from that at 83 ps. For $A^{-} \mathrm{O}_{2}$, a peak at $\sim 0.65-0.8 \mu$ s appears at time zero but disappears within 540 fs. We think that this peak corresponds to the PE signal of the charge-transfer complex $A \cdot \mathrm{O}_{2}^{-}$, even though it is shifted toward earlier arrival time (lower eBE) than the $\mathrm{PE}$ profile of $\mathrm{O}_{2}^{-}$, which should be located at least as low in energy as that of $A \cdot \mathrm{O}_{2}^{-}$. Profiles of $\mathrm{O}_{2}^{-}$with and without a $0.03 \mu \mathrm{s}(\sim 0.3 \mathrm{eV}$ in eBE) shift are illustrated in blue traces in the 0 and 60 fs panels, respectively. This shift can be rationalized by the generation of fast electrons coming off from the vibrationally excited states of the $A \cdot \mathrm{O}_{2}^{-}$complex.

The TD-PE spectra of $A^{-} \mathrm{O}_{4}, A^{-} \mathrm{O}_{6}$, and $A^{-} \mathrm{O}_{8}$ display very similar temporal behaviors represented by the TD-PE spectra of $A^{-} \mathrm{O}_{6}$ in Fig. 3. As for $A^{-} \mathrm{O}_{2}$, a short-lived feature appears at early times $(\sim 0-240 \mathrm{fs})$, but in this case, it is located at $\sim 0.7-0.8 \mu \mathrm{s}$, making it appear as a shoulder to the 83-ps-like component. This could be the signature of either $A \cdot \mathrm{O}_{2}^{-} \cdot\left(\mathrm{O}_{2}\right)_{n-1}$ or $A \cdot \mathrm{O}_{4}^{-} \cdot\left(\mathrm{O}_{2}\right)_{n-2}$. We superimposed the PE profiles of $\mathrm{O}_{2}^{-}$(blue) and $\mathrm{O}_{4}^{-}$(green) as well as the shifted versions of $\mathrm{O}_{2}^{-}$and $\mathrm{O}_{4}^{-}$to offer the same prospective as for the $A^{-} \mathrm{O}_{2}$ case.

The time-dependent photoelectron signals at $t \geqslant 360 \mathrm{fs}$ can be moderately well reconstructed by adding the positive amplitude (production) of $A^{-}$and the negative amplitude (depletion) of the parent, suggesting that the contribution to the positive amplitude is predominantly from $A^{-}$. In order to check the presence of intermediate species, $A^{-}\left(\mathrm{O}_{2}\right)_{n-m}$, $0<m<n$, we simulated the TD-PE profile of $A^{-}\left(\mathrm{O}_{2}\right)_{n}$, where $n=2-4$ at $t \geqslant 360$ fs by adding and subtracting the static PE profiles of $A^{-}\left(\mathrm{O}_{2}\right)_{n}$ (shown on the eBE scale in 


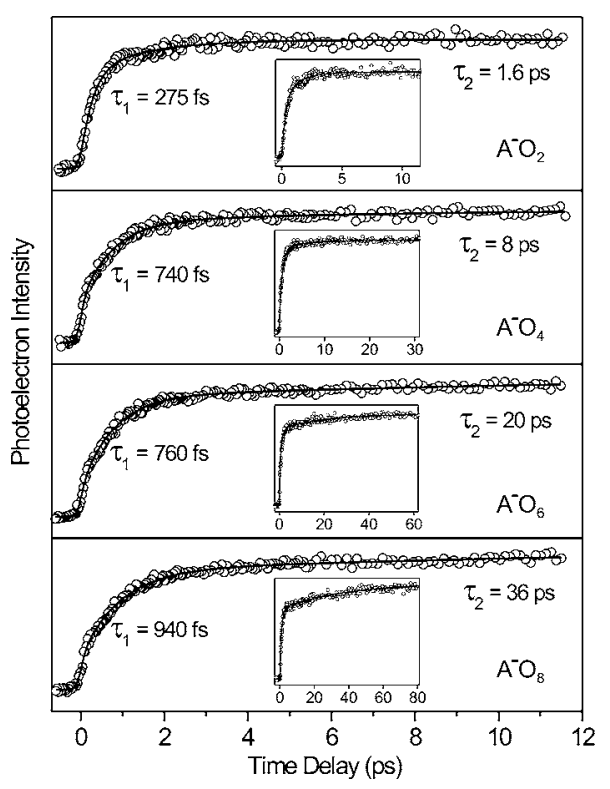

FIG. 4. Femtosecond transients obtained by monitoring the spectral window indicated in Figs. 3(A) and 3(B) for the excitation of $A^{-}\left(\mathrm{O}_{2}\right)_{n}$, where $n$ $=1-4$. The transient of $A^{-} \mathrm{O}_{2}$ is fitted to a biexponential rise function with two time constants $\left(\tau_{1}\right.$ and $\left.\tau_{2}\right)$, while for the other clusters, an additional rise $\left(\tau_{0}<100 \mathrm{fs}\right)$ is included. The fits are shown by solid lines. The inset in the top panel shows an experimental magic angle scan, while all other transients are constructed magic angle scans (see text). Transients of $A^{-}\left(\mathrm{O}_{2}\right)_{n}$, where $n=2-4$, over long time ranges are presented as insets.

Fig. 2). [No simulation using only $A^{-}\left(\mathrm{O}_{2}\right)_{n}$ profiles can account for the early delay time $(t<360 \mathrm{fs})$ TD-PE features at electron time-of-flight below $0.8 \mu \mathrm{s}$.] In our simulation, we forced the intensity of all the possible products $\left[A^{-}\right.$and $\left.A^{-}\left(\mathrm{O}_{2}\right)_{n-m}\right]$ to be positive and that of the parent cluster $\left[A^{-}\left(\mathrm{O}_{2}\right)_{n}\right]$ to be negative. For all clusters, we obtained a reasonable agreement between the simulated and observed TD-PE traces by using only $A^{-}$and the parent with relative photodetachment cross sections $\left(\sigma_{\mathrm{PD}}\left(A^{-}\right) / \sigma_{\mathrm{PD}}\left(A^{-} \mathrm{O}_{2}\right) \sim 2.5\right.$, $\sigma_{\mathrm{PD}}\left(A^{-}\right) / \sigma_{\mathrm{PD}}\left(A^{-} \mathrm{O}_{4}\right) \sim 17, \quad \sigma_{\mathrm{PD}}\left(A^{-}\right) / \sigma_{\mathrm{PD}}\left(A^{-} \mathrm{O}_{6}\right) \sim 10, \quad$ and $\left.\sigma_{\mathrm{PD}}\left(A^{-}\right) / \sigma_{\mathrm{PD}}\left(A^{-} \mathrm{O}_{8}\right) \sim 21\right)$ similar to the rough estimates given above. Such simulations are illustrated in the top two panels of Fig. 3(C) for $A^{-} \mathrm{O}_{2}$ and $A^{-} \mathrm{O}_{6}$.

The success of such simulation is expected for $A^{-} \mathrm{O}_{2}$, since there is no possible intermediate species or alternate channel. In addition, the approximate ratio of $\sigma_{\mathrm{PD}}$ obtained from the simulation yields a more reliable value than that estimated from the ion and PE intensities since we know, within the limit of ion fluctuation, that the equal populations of loss and gain give rise to the two component contributions. For larger clusters, however, the degree to which one can exclude the presence of any intermediate is contingent upon the shape of its PE spectrum. For example, in the third frame it is shown that a contribution from $A^{-} \mathrm{O}_{4}$ can be compensated by a larger negative amplitude of $A^{-} \mathrm{O}_{6}$. However, we found no way to compensate the addition of $\sim 10 \%$ or greater peak height of $A^{-} \mathrm{O}_{2}$ relative to $A^{-}$, suggesting the absence of $A^{-} \mathrm{O}_{2}$ as a significant intermediate or alternate product in this time range. [See Fig. 3(C), bottom for an illustration with $\sim 20 \% A^{-} \mathrm{O}_{2}$ contribution.] The $A^{-} \mathrm{O}_{2}$ fragment ( $\sim 7 \%$ of the height with respect to the $A^{-}$fragment) shown in Fig. 1(C) is consistent with this limit, considering

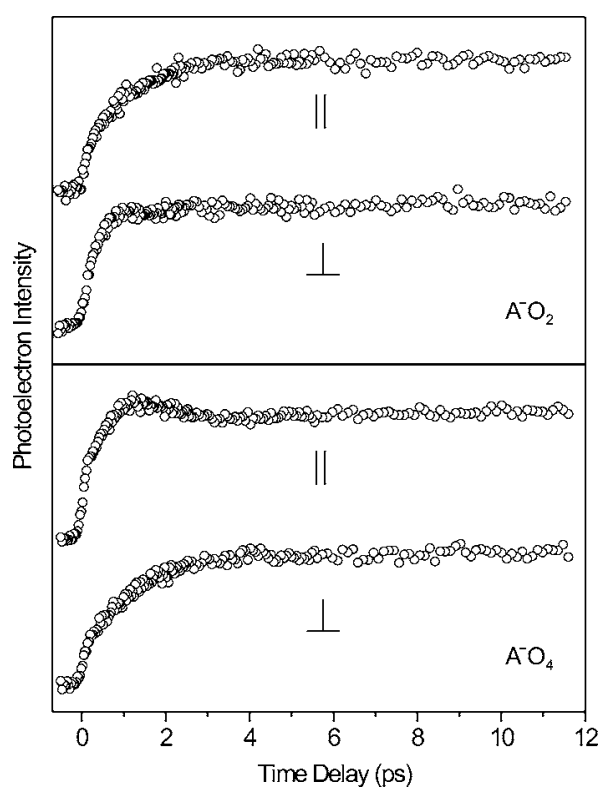

FIG. 5. Polarization-dependent transients at $800 \mathrm{~nm}$. The polarization of the probe pulse was fixed parallel to the photoelectron detection axis, while the polarization of the pump pulse was rotated. $A^{-} \mathrm{O}_{2}$ shows polarizationdependence opposite to the larger clusters, $A^{-}\left(\mathrm{O}_{2}\right)_{n}$ where $n=2-4$, but only $A^{-} \mathrm{O}_{4}$ is provided for comparison.

the relative photodetachment cross section of the two species being similar. These conclusions also apply for the $A^{-} \mathrm{O}_{4}$ and $A^{-} \mathrm{O}_{8}$ parent clusters.

To quantify the temporal behavior illustrated in Fig. 3, we recorded transients by setting the integration window (indicated by the blue box in Fig. 3) at the front edge of the $A^{-}$ $\mathrm{PE}$ profile. The width and position of the boxcar gate were carefully adjusted in order to integrate the PE signature of $A^{-}$ with a minimum contribution from $A^{-}\left(\mathrm{O}_{2}\right)_{n}$. However, $\mathrm{O}_{2}^{-}$, $\mathrm{O}_{4}^{-}, A \cdot \mathrm{O}_{2}^{-} \cdot\left(\mathrm{O}_{2}\right)_{n-1}$, and $A \cdot \mathrm{O}_{4}^{-} \cdot\left(\mathrm{O}_{2}\right)_{n-2}$ may be represented in the transients. For use in the investigation of vectorial dynamics (see below), separate scans with pump and probe polarizations parallel $\left[I_{\|}(t)\right]$ and perpendicular $\left[I_{\perp}(t)\right]$ to each other were carried out for all clusters. Magic angle transients $\left(I_{54.7^{\circ}}\right)$ were constructed from these two polarization scans, $I_{54.7^{\circ}} \propto I_{\|}(t)+2 I_{\perp}(t)$, with the asymptotes of $I_{\|}(t)$ and $I_{\perp}(t)$ normalized to 1. Such transients are shown in Fig. 4 for all clusters studied. In confirmation of the reliability of this procedure, we also obtained the actual magic angle scan for $A^{-} \mathrm{O}_{2}$ (top panel inset of Fig. 4) and $A^{-} \mathrm{O}_{4}$ by rotating the polarization of the pump pulse by $54.7^{\circ}$ with respect to that of the probe pulse. The transients obtained by the two different methods are effectively indistinguishable. (The small residual asymptotic differences between the polarizations that were found without applying normalization are not considered meaningful because of the variation in experimental conditions between the two polarization scans.)

For $A^{-} \mathrm{O}_{2}$, the transients exhibit two apparent rise components with distinct time scales. We fitted the transients to a biexponential growth function, varying time constants $\left(\tau_{1}\right.$ and $\left.\tau_{2}\right)$ and amplitudes with the fixed instrument response time [full width at half maximum $(\mathrm{FWHM})=230 \mathrm{fs}$ ], yielding $\tau_{1} \sim 275 \pm 50 \mathrm{fs}$ and $\tau_{2} \sim 1.6 \pm 0.2 \mathrm{ps}$. For larger clusters, we observed three distinct rise components. The 
fastest component is a rise within our response time $\left(\tau_{0}\right.$ $<100 \mathrm{fs}$ ). We associate the two slower components to $\tau_{1}$ and $\tau_{2}$ of $A^{-} \mathrm{O}_{2}$ (see discussion). The second fastest component has the lifetime $\left(\tau_{1}\right)$ of $\sim 800$ fs (see Fig. 4 ), but the lifetime of the slowest component $\left(\tau_{2}\right)$ increases with cluster size: $8 \pm 1$ ps $\left(A^{-} \mathrm{O}_{4}\right), 20 \pm 2$ ps $\left(A^{-} \mathrm{O}_{6}\right)$, and $36 \pm 4$ ps $\left(A^{-} \mathrm{O}_{8}\right)$. The relative amplitude of the slowest component also increases with cluster size from $A^{-} \mathrm{O}_{4}$ to $A^{-} \mathrm{O}_{8}$, as clearly seen in the long time scans shown as insets in Fig. 4.

The transients significantly depend on the relative polarization of pump and probe pulses. The polarization dependence of $A^{-} \mathrm{O}_{2}$ is opposite to that of $A^{-}\left(\mathrm{O}_{2}\right)_{n}$, where $n$ $=2-4$ (Fig. 5, with $A^{-} \mathrm{O}_{4}$ illustrating the behaviors for larger clusters). From the normalized parallel and perpendicular scans, we constructed the time-dependent anisotropy $r(t)$ :

$$
r(t)=\frac{I_{\|}(t)-I_{\perp}(t)}{I_{\|}(t)+2 I_{\perp}(t)}
$$

The results are plotted in Fig. 6. The solid lines shown with the data are described later (see Sec. IV). As is evident from the relative intensites of the transients of Fig. 5, $r(t)$ of $A^{-} \mathrm{O}_{2}$ is of opposite sign to that of the larger clusters at early time. The anisotropy of $A^{-} \mathrm{O}_{2}$ starts at a negative value and approaches zero, whereas the anisotropy of larger clusters starts at positive values and decreases. The time scale of the decay toward zero increases as the cluster size increases, and the coherence time $\left(\tau_{c}\right)$, taken as the time required for the anisotropy to fall to one-fourth of its initial value, ${ }^{9}$ is estimated and indicated in Fig. 6: 1.7 ps $\left(A^{-} \mathrm{O}_{2}\right), 2.2$ ps $\left(A^{-} \mathrm{O}_{4}\right), 3.0$ ps $\left(A^{-} \mathrm{O}_{6}\right)$, and $3.0 \mathrm{ps}\left(A^{-} \mathrm{O}_{8}\right)$.

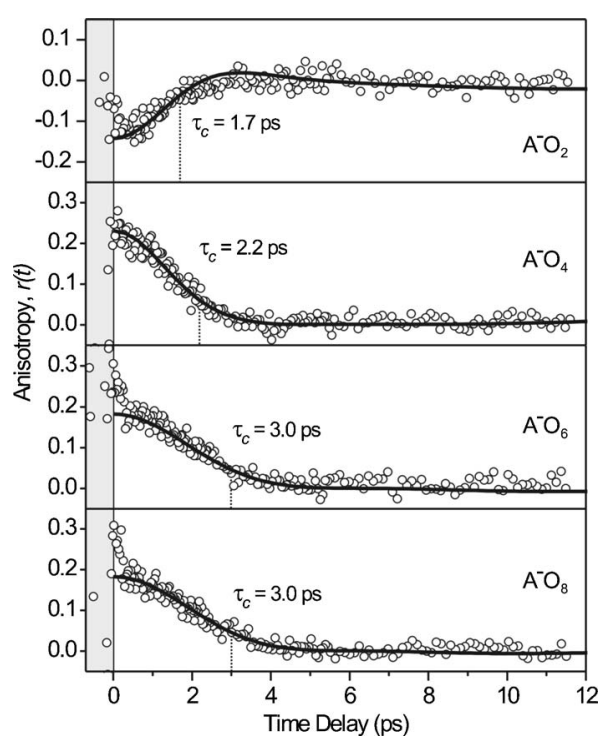

FIG. 6. Time-dependent anisotropy decay $r(t)=\left(I_{\|}-I_{\perp}\right) /\left(I_{\|}+2 I_{\perp}\right)$ obtained from parallel $\left(I_{\|}\right)$and perpendicular $\left(I_{\perp}\right)$ transient scans normalized to give $r(t)=0$ at long time. The estimated coherence times $\left(\tau_{c}\right)$ are indicated (see text). Solid lines are the fits using the hypothesized structures in Fig. 7 as described in the text. The grey shading indicates time $t<0$ for which $I_{\|}$and $I_{\perp}$ are small and $r(t)$ becomes erratic.

\section{DISCUSSION}

Based on the experimental observations discussed above, the mechanism for the photodissociation of $A^{-}\left(\mathrm{O}_{2}\right)_{n}$, where $n=1-4$, is described below, highlighting the elementary steps of electron transfer and ultrafast electron recombination:

$$
\begin{gathered}
A^{-} \mathrm{O}_{2} \stackrel{h \nu}{\longrightarrow}\left[A \cdot \mathrm{O}_{2}^{-}\right]^{*} \stackrel{\text { ER }}{\longrightarrow} A^{-} \mathrm{O}_{2} \rightarrow A^{-}+\mathrm{O}_{2} \\
A^{-}\left(\mathrm{O}_{2}\right)_{n} \stackrel{h \nu}{\longrightarrow}\left[A \cdot \mathrm{O}_{4}^{-} \cdot\left(\mathrm{O}_{2}\right)_{n-2}\right]^{*} \mathrm{O}_{2}^{-}, \\
\stackrel{\mathrm{ER}}{\longrightarrow} A^{-}\left(\mathrm{O}_{2}\right)_{n} \rightarrow\left\{\begin{array}{c}
A^{-}\left(\mathrm{O}_{2}\right)_{n-1}+\mathrm{O}_{2} \\
A^{-}\left(\mathrm{O}_{2}\right)_{n-2}+2 \mathrm{O}_{2} \\
\vdots \\
A^{-}+\left(\mathrm{O}_{2}\right)_{n}
\end{array}\right\} \rightarrow \cdots \rightarrow A^{-}+n \cdot \mathrm{O}_{2} \\
\stackrel{D}{\longrightarrow} A+\mathrm{O}_{4}^{-}+(n-2) \cdot \mathrm{O}_{2} .
\end{gathered}
$$

The primary processes are similar in nature to those of oxygen clusters. ${ }^{8,9,13}$ Here, $h \nu$ is the $800 \mathrm{~nm}$ pump photon energy, $A \cdot \mathrm{O}_{2}^{-}$and $A \cdot \mathrm{O}_{4}^{-} \cdot\left(\mathrm{O}_{2}\right)_{n-2}$ are the charge-transfer complexes, and ER and $D$ denote electron recombination and dissociation, respectively. As discussed earlier in Sec. III, the metastable patterns and photoelectron spectra suggest that the excess electron is located in azobenzene $(A)$, and oxygen molecules are weakly bound to $A^{-}$. The ab initio geometry calculation of the trans-azobenzene anion by the $\mathrm{UB} 3 \mathrm{LYP} / 6-311 \mathrm{G}(d, p)$ method suggests that the excess 
electron is localized in the $\mathrm{N}-\mathrm{N}$ double bond. ${ }^{20} \mathrm{~A}$ small amount $(\sim 0.1 \%)$ of $\mathrm{O}_{4}^{-}$was detected from metastable $\left(A \cdot \mathrm{O}_{4}\right)^{-}$, compared to the $A^{-}$intensity of $\sim 2 \%$, which suggests that a part of the population of $\left(A \cdot \mathrm{O}_{4}\right)^{-}$exists in the form of $\mathrm{A} \cdot \mathrm{O}_{4}^{-}$.

\section{A. Scalar dynamics}

From the photofragmentation study, it is inferred that when an oxygen molecule is attached to $A^{-}$, there exists a charge-transfer band around $1.55 \mathrm{eV}(800 \mathrm{~nm})$ above the ground state of the $A^{-}\left(\mathrm{O}_{2}\right)_{n}$ anion. Species with no oxygen molecules such as $A_{2}^{-}$, as shown in Fig. 1(C), and $A_{3}^{-}$did not dissociate to a significant extent by $800 \mathrm{~nm}$ photon, even though the binding energy between $A^{-}$and the neutral $A$ is similar to the binding energy between $A^{-}$and $\mathrm{O}_{2}{ }^{21}$ The small intensities of $\mathrm{O}_{2}^{-}$and $\mathrm{O}_{4}^{-}$fragments generated from $A^{-} \mathrm{O}_{2}$ and $A^{-} \mathrm{O}_{4}$ upon $800 \mathrm{~nm}$ irradiation can be explained by the dissociation of the charge-transfer complexes $A \cdot \mathrm{O}_{2}^{-}$and $A \cdot \mathrm{O}_{4}^{-}$, respectively, during their subpicosecond lifetimes. However, the predominant intensity of $A^{-}$indicates that the excess electron tends to transfer back to $A$ prior to dissociation. The fact that contributions of $\mathrm{O}_{2}^{-}$and $\mathrm{O}_{4}^{-}$are not identified in the TD-PE spectra is understood because of their low yields and the near coincidence of their PE spectra with that of $A^{-}$.

For $A^{-} \mathrm{O}_{2}$, upon $800 \mathrm{~nm}$ excitation, the excess electron migrates from $A^{-}$to $\mathrm{O}_{2}$, forming $A \cdot \mathrm{O}_{2}^{-}$followed by ultrafast electron recombination and dissociation. The formation of $A \cdot \mathrm{O}_{2}^{-}$is evident in the TD-PE spectra of $A^{-} \mathrm{O}_{2}$. As discussed in the previous section, the peak at $\sim 0.7 \mu$ s time-of-flight is assigned to the PE detached from excited vibrational states of $A \cdot \mathrm{O}_{2}^{-}$. For all larger clusters, however, we think that the charge-transfer complex in the transition state forms a specific ionic species, $A \cdot \mathrm{O}_{4}^{-} \cdot\left(\mathrm{O}_{2}\right)_{n-2}$ rather than $A \cdot \mathrm{O}_{2}^{-} \cdot\left(\mathrm{O}_{2}\right)_{n-1}$ based on two experimental observations dealing with a $\mathrm{O}_{4}^{-}$ signature. First, $\mathrm{O}_{4}^{-}$but not $\mathrm{O}_{2}^{-}$was generated from the photodissoication of $A^{-} \mathrm{O}_{4}$ [Fig. $\left.1(\mathrm{C})\right]$. For $A^{-} \mathrm{O}_{6}$ and $A^{-} \mathrm{O}_{8}$, we did not observe any negatively charged oxygen clusters as fragment, but this can be due to the small intensity of parent ions [Fig. 1(A)] and insufficient detection sensitivity. Second, we found two distinct types of early-delay-time PE signal behaviors which are evident in Figs. 3(A) and 3(B), in the position of the shoulder feature, and in Fig. 4, in the prominence of the instantaneous rise component. The transition between the two behaviors occurs upon going from $A^{-} \mathrm{O}_{2}$ to $A^{-} \mathrm{O}_{4}$. The relationship between these behaviors and the evidence for a $\mathrm{O}_{4}$ core formation in static measurements will be discussed later.

In the absence of knowledge of the absorption spectra of $A^{-}\left(\mathrm{O}_{2}\right)_{n}$, one cannot definitively exclude the possibility that $\mathrm{O}_{2}$ perturbs the electronic state of $A^{-}$such that an excited state of $A^{-}$becomes accessible at $800 \mathrm{~nm}$, without invoking electron transfer (ET) and recombination processes. A similar situation has been discussed by Li et al. for the case of $\mathrm{O}_{4}^{-} \cdot \mathrm{O}_{2}$ dissociation. ${ }^{4}$ However, the observations of $\mathrm{O}_{4}^{-}$and $\mathrm{O}_{2}^{-}$photofragments and the lack of dissociation of clusters without $\mathrm{O}_{2}$ reported here, as well as the fact that the excitation energy $(1.55 \mathrm{eV})$ matches the expected energy of a charge-transfer band, ${ }^{13}$ i.e., between AEA and VDE of $A^{-}\left(\mathrm{O}_{2}\right)_{n}$, lead us to favor a photoinduced intermolecular electron transfer followed by an ultrafast recombination as a more appropriate reaction mechanism for the absorption of $800 \mathrm{~nm}$ light, similar to the mechanism discussed for both neutral and anionic clusters. ${ }^{8,9,22}$ Especially, we have found that oxygen plays a significant role in the photoinduced electron transfer at $800 \mathrm{~nm}$ and examined this phenomenon in solvated oxygen anions: For example, we observed that $\mathrm{O}_{4}^{-} X$, $X=\mathrm{Xe}, \mathrm{N}_{2}$, and $\mathrm{N}_{2} \mathrm{O}$, does not dissociate while $\mathrm{O}_{4}^{-} \mathrm{O}_{2}$ dissociates at $800 \mathrm{~nm}^{9}$

For $A^{-} \mathrm{O}_{2}, A^{-}$rises via two different channels. The fast channel reflects the direct bond dissociation along the $A^{-}-\mathrm{O}_{2}$ coordinate. The initial excitation energy is efficiently used to break the bond between $A^{-}$and $\mathrm{O}_{2}$, and the energy dissipation to other modes is not as effective. The time constant of the fast component depends on the rates of ER and the subsequent motion of $\mathrm{O}_{2}$ along the $A^{-}-\mathrm{O}_{2}$ coordinate, as described in Eq. (2a). The rate of ER is approximated from the decay of $A \cdot \mathrm{O}_{2}^{-}$, which is comparable to our instrument response time but not quantified. Since the pump photon energy $(1.55 \mathrm{eV})$ is much larger than the bond energy of $A^{-}-\mathrm{O}_{2}(\sim 0.2 \mathrm{eV})$, the direct dissociation can occur on the subpicosecond time scale. The slow component depicts the evaporative dissociation of another fraction of the $A^{-} \mathrm{O}_{2}$ population, in which the vibrational energy released upon recombination couples less effectively to the reaction coordinate. The rate depends on the degree of involvement of all vibrational modes of the complex. Additional dissociation channels may exist involving a more extensive intramolecular vibrational energy redistribution (IVR) and much lower rates, but these channels would not be detectable in our timedelay window $(0-83 \mathrm{ps})$. The bifurcation of the parent population into different dissociation channels can be envisioned as reflecting a structural inhomogeneity in the bonding geometry.

For $A^{-}\left(\mathrm{O}_{2}\right)_{n}$, where $n=2-4$, three distinct components are observed in the transients. The instantaneous rise component is attributed to the formation of the charge-transfer transient complex $A \cdot \mathrm{O}_{4}^{-} \cdot\left(\mathrm{O}_{2}\right)_{n-2}$. The corresponding component $\left(A \cdot \mathrm{O}_{2}^{-}\right)$is not evident in the transient of $A^{-} \mathrm{O}_{2}$ because the $A^{-}$ rise itself has a very fast temporal onset, and possibly also because of the displacement of the $A \cdot \mathrm{O}_{2}^{-} \mathrm{PE}$ signal from the boxcar gate position [Fig. 3(A)]. The inability to distinguish an instantaneous rise in the $A^{-} \mathrm{O}_{2}$ transient is demonstrated by the fact that the fit shown in Fig. 4 and a fit with the addition of a $20 \%$ instantaneous rise component are equally satisfactory. The two time constants derived from the transients are only weakly affected by the presence of the additional instantaneous component. On the other hand, the very clear instantaneous rise component in $A^{-}\left(\mathrm{O}_{2}\right)_{n}$, where $n$ $=2-4$, indicates that the PE of the charge-transfer complex lies in the boxcar gate. This is expected for $A \cdot \mathrm{O}_{4}^{-} \cdot\left(\mathrm{O}_{2}\right)_{n-2}$ since the $\mathrm{PE}$ spectra of $\mathrm{O}_{4}^{-}$and $A^{-}$are similar and the solvent molecules around $\mathrm{O}_{4}^{-}$do not alter the $\mathrm{PE}$ spectrum of $\mathrm{O}_{4}^{-}$as observed in other similar clusters. ${ }^{9}$ That the charge-transfer complex for these clusters is different from that of the $A^{-} \mathrm{O}_{2}$ case is evident from the TD-PE spectra.

The second fast component in the transients of $A^{-}\left(\mathrm{O}_{2}\right)_{n}$, 
where $n=2-4$, has a time constant $\left(\tau_{1}\right)$ of $\sim 800 \mathrm{fs}$. We interpret this component as analogous to the fast rise in the transient of $A^{-} \mathrm{O}_{2}$, i.e., as electron recombination/direct dissociation of the parent cluster. While gating was set up to monitor the rise of bear $A^{-}$, the observed rate is the effective rate constant for ER and the parallel dissociation channels [Eq. (2b)]. The increase in $\tau_{1}$ upon going from $A^{-} \mathrm{O}_{2}$ to $A^{-}\left(\mathrm{O}_{2}\right)_{n}$, where $n=2-4$, may possibly reflect the formation of $A \cdot \mathrm{O}_{4}^{-} \cdot\left(\mathrm{O}_{2}\right)_{n-2}$ which has a stronger electron binding than $A \cdot \mathrm{O}_{2}^{-}$and thus prolongs the electron recombination process. The time scale of $\tau_{1}$ in the transient of $A^{-} \mathrm{O}_{8}$ is still in the subpicosecond regime, showing that the observed rise of $A^{-}$ in this time range is via a single-bond breakage between $A^{-}$ and the conglomerated $\left(\mathrm{O}_{2}\right)_{n}$, rather than a stepwise evaporation of oxygen molecules. The fact that the fast component is not strongly affected by additional oxygen molecules lends support to our hypothesis that the rate determining step is ER rather than the subsequent parallel channels of dissociation [Eq. (2b)]. If the latter determine the rate, we should see a distinct cluster size dependence.

As in the case of $A^{-} \mathrm{O}_{2}$, based on our TD-PE analyses and the single-exponential-type rise of the $\tau_{2}$ component, we also believe that the slow channel is via a single-bond breakage like the $\tau_{1}$ process. Thus, the existence of the $\tau_{2}$ component is believed to reflect the structural variation within the clusters, which constitutes a bifurcation between the geometries of optimal recombination/dissociation and residual geometries in a manner reminiscent of base-pair gating of electron transfer through DNA. ${ }^{23}$ Ideally aligned $A^{-}\left(\mathrm{O}_{2}\right)_{n}$ undergoes rapid electron recombination and concomitant dissociation, while the recombination and dissociation may be delayed in misaligned complexes. An increasing entropy factor in larger clusters dictates the ratio between the ideal and the remaining cases, which is shown in the observed trend of relative contribution by the $\tau_{2}$ component. $A^{-} \mathrm{O}_{2}$ may have a completely different geometry, which results in the deviation from the observed trend. This aspect is highlighted in the time-resolved anisotropy (see below). In this picture, the variation in $\tau_{2}(\sim 8-36 \mathrm{ps})$ results from a quasistatistical dissociation as in the case of $A^{-} \mathrm{O}_{2}$. As noted earlier, a small $A^{-} \mathrm{O}_{2}$ intensity in the fragment mass spectra [Fig. 1(C)] could be produced by a parallel channel not identified in our timeresolved data, consistent with the sensitivity limit shown in Fig. 3(C).

\section{B. Vectorial dynamics}

As illustrated in Figs. 5 and 6, the transients depend on the orientation of pump and probe polarizations, which indicates time-dependent vectorial dynamics of the PE detachment transition moment. The anisotropy is related to the angle $\alpha$ between the excitation and probe transition dipole moments by ${ }^{24} r(t)=0.4\left\langle P_{2}(\cos \alpha(t))\right\rangle$ where $P_{2}$ is the second order Legendre polynomial, $P_{2}(x)=\frac{1}{2}\left(3 x^{2}-1\right)$, and the brackets indicate an ensemble average. The time dependence of $r(t)$ reflects the reorientation of probe dipoles of molecules undergoing rotational motion, but at $t=0$ the value is determined solely by the static directions of the two transitions in the molecular frame, which we assume are the same for all
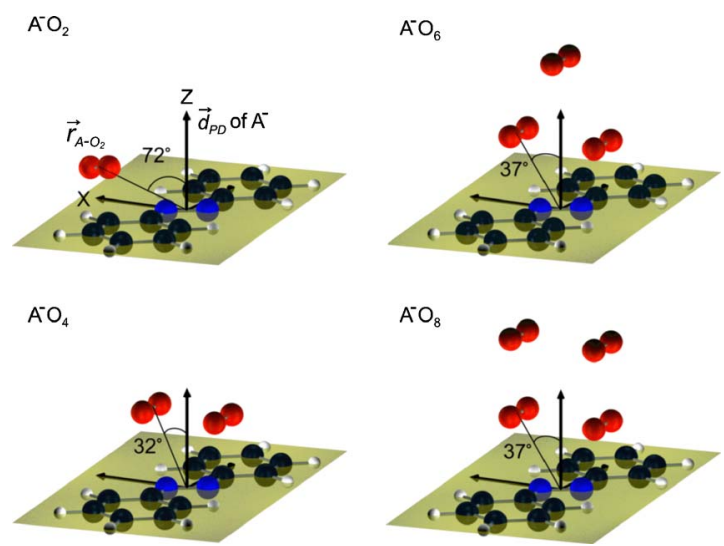

FIG. 7. (Color online) Proposed structures for $A^{-}\left(\mathrm{O}_{2}\right)_{n}$, where $n=1-4$. The midpoints of $\mathrm{O}_{2}$ molecules lie on the $X Z$ plane. The deduced transition dipole moment for the photodetachment process of $A^{-}\left(\vec{d}_{\mathrm{PD}}\right)$ is along the $z$ axis. Angles between $\vec{r}_{A^{-} \mathrm{O}_{2}}$ and $\bar{d}_{\mathrm{PD}}$ are indicated.

molecules of a given stoichiometry. Based on our mechanism that pump-pulse excitation involves ET from $A^{-}$to $\mathrm{O}_{2}$ or $\mathrm{O}_{4}$, we can assume that the excitation transition dipole moment lies along the direction of the electron motion. From the anisotropy values at time zero (Fig. 6), the angle $\alpha(0)$ between this excitation transition moment and the transition dipole moment of the photodetachment process $\left(\vec{d}_{\mathrm{PD}}\right)$ of $A^{-}$ can therefore be determined from $\cos \alpha(0)=\sqrt{\frac{1}{3}(5 r(0)+1)}$. Ignoring for the moment the very early time behavior $(t<400 \mathrm{fs})$ of $r(t)$ and extrapolating the ps-scale trend back to $t=0$, we estimate the following values for $r(0)$ of $\vec{d}_{\mathrm{PD}}$ of $A^{-}$from $A^{-}\left(\mathrm{O}_{2}\right)_{n}:-0.14,0.23,0.18$, and 0.18 for $n=1,2,3$, and 4 , respectively. Thus, assuming single, well-defined pump and probe transition moment vectors, one finds for $A^{-} \mathrm{O}_{2}$ an angle between $\vec{r}_{A^{-} \mathrm{O}_{2}}$ and $\vec{d}_{\mathrm{PD}}$ of $72^{\circ}$ and for $A^{-}\left(\mathrm{O}_{2}\right)_{n}$, where $n=2-4$, an angle between the electron motion and $\vec{d}_{\mathrm{PD}}$ ranging from $32^{\circ}$ to $37^{\circ}$.

Given that the probe process (photodetachment of $A^{-}$) is the same in each case and presuming that the excess electron rests on the $\mathrm{N}-\mathrm{N}$ bond for each parent, these results show that the position of $\mathrm{O}_{2}$ in $A^{-} \mathrm{O}_{2}$ is markedly different from that of the $\mathrm{O}_{4}$ core in $A^{-}\left(\mathrm{O}_{2}\right)_{n}$, where $n=2-4$. For example, a plausible interpretation of the data is represented by the structures illustrated in Fig. 7, with $\mathrm{O}_{2}$ in $A^{-} \mathrm{O}_{2}$ located near the plane of the azobenzene molecule, the oxygen molecules in $A^{-}\left(\mathrm{O}_{2}\right)_{n}$, where $n=2-4$, forming an oxygen cluster near the $z$ axis (perpendicular to the plane of $A^{-}$), and $\vec{d}_{\mathrm{PD}}$ of $A^{-}$ along that axis. In this picture, the electron jumps from the $\mathrm{N}-\mathrm{N}$ bond to one of the adjacent $\mathrm{O}_{2}$ molecules which is located, on average, in the direction indicated, with a second $\mathrm{O}_{2}$ in place to complete the formation of the $\mathrm{O}_{4}^{-}$core.

In the absorption process, the polarized $800 \mathrm{~nm}$ pulses produce a well-defined spatial alignment of the chargetransfer state population. After the recombination and dissociation, the rotational motion of the $A^{-}$ensemble with a distribution of angular velocities causes the initial alignment to dephase and the anisotropy to decay. For all clusters, the anisotropy approaches its asymptotic value within a few picoseconds. The time scale of the anisotropy decay can be 
related to the cluster structure, the inertial motion of the parent clusters at finite temperature, and to any torque applied to $A^{-}$during dissociation. ${ }^{10}$ Under the simplifying assumption of the dissociation of $A^{-}\left(\mathrm{O}_{2}\right)_{n}$ with no torque, the instantaneous angular velocity of the $A^{-}$immediately after dissociation will be the same as the angular velocity of the parent by the conservation of angular momentum. The calculation of the rotational dephasing of the parent clusters may therefore serve as an approximation of the fragment $A^{-}$anisotropy decay.

Such calculations were carried out for each cluster based on the structures and transition dipole directions of Fig. 7 and with rotational temperatures adjusted to give values of $\tau_{c}$ matching the experiment. The quantum mechanical asymmetric top rotational coherence theory ${ }^{25}$ was used for these calculations, implemented by a program allowing arbitrary directions of the transition dipoles. ${ }^{26}$ The results are shown as the solid lines plotted with the data in Fig. 6. The temperatures found through this procedure fell in a reasonable range of $\sim 80 \mathrm{~K}$ for $A^{-} \mathrm{O}_{2}$ to $200 \mathrm{~K}$ for $A^{-} \mathrm{O}_{8}$. At times greater than $400 \mathrm{fs}$, the shapes of the decays are in generally good agreement with the theoretical models as well.

At very early times, the anisotropies of three of the four clusters studied show a deviation from the smooth trend predicted from the rotational motion of $A^{-}$only. Such behavior might be caused by a shift of only $\sim 30 \mathrm{fs}$ in the time axis between measurements of perpendicular and parallel transients, so the values must be viewed with caution. However, a real change in anisotropy is easily understood in the context of the dynamics described by Eq. (2), since the first signal detected is that of the short-lived charge-transfer state rather than $A^{-}$, and these two species need not have the same direction for their PD transition dipoles. The observed anisotropy at short delay times is then a signal-intensity-weighted average of the $r(0)$ values (assuming virtually no rotational motion in the first $500 \mathrm{fs}$ ) of the two contributing populations, as determined by their respective PD transition dipoles. As the ET state decays and $A^{-}$rises, the anisotropy is expected to approach the $r(0)$ value of $A^{-}$nonexponentially, and the time scale for the change observed in Fig. 6 is compatible with the population dynamics derived from the transients of Fig. 4. The fact that $r(t)$ of $A^{-} \mathrm{O}_{4}$ shows no deviation at early times means that $\vec{d}_{\mathrm{PD}}$ of $A^{-} \mathrm{O}_{4}$ is the same as $\vec{d}_{\mathrm{PD}}$ of $A^{-}$, i.e., perpendicular to the $\mathrm{O}_{4}$ plane in the proposed structure. The deviation in the $r(t)$ of $A^{-} \mathrm{O}_{2}$ below $400 \mathrm{fs}$ is evidence of the ET state contribution that is not discernible in the magic angle data but is expected based on consideration of the TD-PE spectra.

Finally, it needs to be re-emphasized that the deduced solvation configuration exhibited in $A^{-}\left(\mathrm{O}_{2}\right)_{n}$ is unique among other analogous clusters such as $\mathrm{I}_{2}^{-}\left(\mathrm{CO}_{2}\right)_{n}$ and $\mathrm{I}_{2}^{-} \mathrm{Ar}_{n}{ }^{27-29}$ Delaney et al. suggested that all four $\mathrm{CO}_{2}$ molecules are in contact with $\mathrm{I}_{2}^{-}$in $\mathrm{I}_{2}^{-}\left(\mathrm{CO}_{2}\right)_{4}$ : Three carbon dioxide molecules are around the waste of $\mathrm{I}_{2}^{-}$and the fourth one is attached to one of the I atoms, causing asymmetric charge distribution along the $\mathrm{I}_{2}$ axis. ${ }^{28}$ If $A^{-}\left(\mathrm{O}_{2}\right)_{4}$ had a similar solvent arrangement as $\mathrm{I}_{2}^{-}\left(\mathrm{CO}_{2}\right)_{4}$, it is not expected that all four $\mathrm{O}_{2}$ molecules could be stripped off in less than one picosecond. Instead, we propose that $\mathrm{O}_{2}$ molecules cluster together based on the tendency for quasi- $\mathrm{O}_{4}$-core formation in the presence of an extra electron, and this cluster sits on $A^{-}$forming a single bond between $A^{-}$and $\left(\mathrm{O}_{2}\right)_{n}$.

One might speculate that the formation of the conglomerated $\left(\mathrm{O}_{2}\right)_{n}$ is related to the tendency to form a dimeric $\mathrm{O}_{4}^{-}$ core, ${ }^{3,8,9,30}$ and we in fact found a few indirect indications. First, no metastable form of $A^{-} \mathrm{O}_{2}$ was detected for $A^{-} \mathrm{O}_{6}$ and $A^{-} \mathrm{O}_{8}$ while the intensity of $A^{-} \mathrm{O}_{4}$ was quite significant [Fig. 1(B)]. The absence of $A^{-} \mathrm{O}_{2}$ suggests that the second oxygen in $A^{-} \mathrm{O}_{4}$ is more tightly bound than $\mathrm{O}_{2}$ in $A^{-} \mathrm{O}_{2}$. Second, $\mathrm{O}_{4}^{-}$ but not $\mathrm{O}_{2}^{-}$was detected in the photodissociation of $A^{-} \mathrm{O}_{4}$. Third, the qualitative change in the TD-PE spectra at short time from $A^{-} \mathrm{O}_{2}$ to $A^{-}\left(\mathrm{O}_{2}\right)_{n}$, where $n=2-4$ [Figs. 3(A) and $3(\mathrm{~B})]$ tends to indicate that the charge-transfer complex is not the form of $A \cdot \mathrm{O}_{2}^{-} \cdot\left(\mathrm{O}_{2}\right)_{n-1}$ and thus may reasonably be assigned as $A \cdot \mathrm{O}_{4}^{-} \cdot\left(\mathrm{O}_{2}\right)_{n-2}$. Fourth, our anisotropy study also indicates the complete change in the relative position of oxygen molecules from $A^{-} \mathrm{O}_{2}$ to $A^{-} \mathrm{O}_{4}$, which may be understood to be a consequence of the quasi- $\mathrm{O}_{4}$-core formation.

\section{CONCLUSIONS}

In the present work, we have elucidated the dissociation dynamics of $A^{-}\left(\mathrm{O}_{2}\right)_{n}$, where $n=1-4$, involving photoinduced electron transfer, ultrafast electron recombination, bond rupture, and intramolecular vibrational energy redistribution. The evolution of these elementary processes as a function of cluster size was investigated by femtosecond time-resolved photoelectron spectroscopy. Our femtosecond time resolution with the capability of following the vectorial dynamics allows us to develop the following interpretation of the structural evolution of $A^{-}\left(\mathrm{O}_{2}\right)_{n}$ upon going from $A^{-} \mathrm{O}_{2}$ to $A^{-} \mathrm{O}_{8}: \mathrm{O}_{2}$ in $A^{-} \mathrm{O}_{2}$ is located close to the plane of $A^{-}$, whereas conglomerated $\left(\mathrm{O}_{2}\right)_{n}$, where $n=2-4$, in larger clusters sits above the plane of $A^{-}$near the central $\mathrm{N}-\mathrm{N}$ bond.

\section{ACKNOWLEDGMENTS}

This work was supported by the National Science Foundation and the Air Force Office of Scientific Research.

${ }^{1}$ A. W. Castleman, Jr. and K. H. Bowen, J. Phys. Chem. 100, 12911 (1996), and references therein.

${ }^{2}$ P.-Y. Cheng, J. S. Baskin, and A. H. Zewail, Proc. Natl. Acad. Sci. U.S.A. (in press), and references therein.

${ }^{3}$ M. J. DeLuca, C. C. Han, and M. A. Johnson, J. Chem. Phys. 93, 268 (1990).

${ }^{4}$ R. Li, K. A. Hanold, M. C. Garner, A. K. Luong, and R. E. Continetti, Faraday Discuss. 108, 115 (1997), and references therein.

${ }^{5}$ M. Nadal, S. Nandi, P. Wenthold, J. Kim, L. J. Andersen, Y. Ozaki, D. W. Boo, and W. C. Lineberger, in Femtochemistry and Femtobiology: Ultrafast Reaction Dynamics at Atomic-Scale Resolution, Nobel Symposium 101, edited by V. Sundström (Imperial College, London, 1997), and references therein.

${ }^{6}$ D. H. Paik, I. R. Lee, D. S. Yang, J. S. Baskin, and A. H. Zewail, Science 306, 672 (2004).

${ }^{7}$ A. Stolow, A. E. Bragg, and D. M. Neumark, Chem. Rev. (Washington, D.C.) 104, 1719 (2004), and references therein.

${ }^{8}$ N. J. Kim, D. H. Paik, and A. H. Zewail, J. Chem. Phys. 118, 6930 (2003).

${ }^{9}$ D. H. Paik, N. J. Kim, and A. H. Zewail, J. Chem. Phys. 118, 6923 (2003).

${ }^{10}$ J. S. Baskin and A. H. Zewail, J. Phys. Chem. 98, 3337 (1994).

${ }^{11}$ J. S. Baskin and A. H. Zewail, J. Phys. Chem. A 105, 3680 (2001), and references therein. 
${ }^{12}$ P. M. Felker and A. H. Zewail, in Femtosecond Chemistry, edited by J. Manz and L. Wöste (VCH Publishing, New York, 1994), Vol. 1, p. 193, and references therein.

${ }^{13}$ D. H. Paik, T. M. Bernhardt, N. J. Kim, and A. H. Zewail, J. Chem. Phys. 115, $612(2001)$.

${ }^{14}$ R. Weinkauf, K. Walter, C. Weickhardt, U. Boesl, and E. W. Schlag, Z. Naturforsch., A: Phys. Sci. 44a, 1219 (1989).

${ }^{15}$ P. Kruit and F. H. Read, J. Phys. E 16, 313 (1983).

${ }^{16}$ S. R. Gandhi and R. B. Bernstein, Chem. Phys. 105, 423 (1986).

${ }^{17}$ S. Pedersen and A. H. Zewail, Mol. Phys. 89, 1455 (1996).

${ }^{18}$ S. Ingemann, R. H. Fokkens, and N. M. M. Nibbering, J. Org. Chem. 56, 607 (1991).

${ }^{19}$ S. Ingemann, K. V. Larsen, K. B. Haugshøj, and O. Hammerich, Acta Chem. Scand. 43, 981 (1989).

${ }^{20}$ M. J. Frisch, G. W. Trucks, H. B. Schlegel et al., GAUSSIAN 98, Revision A.9, Gaussian, Inc., Pittsburgh, PA, 1998.

${ }^{21}$ The PE spectra of $A_{2}^{-}$and $A_{3}^{-}$resemble the PE spectra of $A^{-} O_{2}$ with similar electron binding energies for $A_{2}^{-}(\mathrm{AEA}=1.2 \mathrm{eV}$ and $\mathrm{VDE}$
$=1.8 \mathrm{eV})$ and for $A_{3}^{-}(\mathrm{AEA}=1.3 \mathrm{eV}$ and $\mathrm{VDE}=1.9 \mathrm{eV})$.

${ }^{22}$ D. Zhong, T. M. Bernhardt, and A. H. Zewail, J. Phys. Chem. A 103, 10093 (1999).

${ }^{23}$ C. Z. Wan, T. Fiebig, S. O. Kelley, C. R. Treadway, J. K. Barton, and A. H. Zewail, Proc. Natl. Acad. Sci. U.S.A. 96, 6014 (1999).

${ }^{24}$ See, for example, G. R. Fleming, Chemical Applications of Ultrafast Spectroscopy (Oxford University Press, New York, 1986).

${ }^{25}$ P. M. Felker and A. H. Zewail, J. Chem. Phys. 86, 2460 (1987).

${ }^{26}$ J. S. Baskin, Ph.D. thesis, California Institute of Technology, 1990.

${ }^{27}$ A. V. Davis, R. Wester, A. E. Bragg, and D. M. Neumark, J. Chem. Phys. 117, 4282 (2002).

${ }^{28}$ N. Delaney, J. Faeder, P. E. Maslen, and R. Parson, J. Phys. Chem. A 101, 8147 (1997).

${ }^{29}$ V. Vorsa, P. J. Campagnola, S. Nandi, M. Larsson, and W. C. Lineberger, J. Chem. Phys. 105, 2298 (1996).

${ }^{30}$ C. R. Sherwood, K. A. Hanold, M. C. Garner, K. M. Strong, and R. E. Continetti, J. Chem. Phys. 105, 10803 (1996). 